\title{
Responsabilidad social y ambiental en las pequeñas y medianas empresas (PYMES)
}

María Susana Ramos Aranda Universidad Santo Tomas

Colombia

Eduardo Augusto Duque Cuesta Fundación Universitaria del Área Andina Colombia 


\title{
Responsabilidad social y ambiental en las pequeñas y medianas empresas (PYMES)
}

\author{
María Susana Ramos Aranda1 ${ }^{\text {iD }}$, Eduardo Augusto Duque Cuesta ${ }^{2}$ iD \\ 1 Universidad Santo Tomas, Colombia \\ 2 Fundación Universitaria del Área Andina, Colombia
}

\section{RESUMEN}

El presente documento analiza la responsabilidad social y ambiental de las pequeñas y medianas empresas (PYMES) de Bogotá con el fin de identificar las prioridades de estas organizaciones en relación con su contribución al mejoramiento ambiental y social de la ciudad. La responsabilidad social y ambiental empresarial debe entenderse como aquellas actividades responsables que van más allá del cumplimiento de las normas y la legislación empresarial. Independientemente del tamaño de la empresa, la falta de una eficiente gestión social y ambiental puede desencadenar problemáticas como el uso indebido de los recursos naturales, contaminación ambiental, empleos de baja calidad entre otros. A partir de la aplicación de instrumentos para la recolección de información, se encontró que las PYMES de la ciudad de Bogotá conocen el tema de la Responsabilidad Social y Ambiental, y realizan acciones que consideran responsables ambiental y socialmente. Sin embargo, no miden los impactos que su operación y comercialización generan, ni tampoco miden el impacto de sus actividades de responsabilidad social y ambiental.

Palabras clave: Responsabilidad social; Responsabilidad ambiental; PYMES; Gestión. JEL: M10, M14, M19

\section{Social and environmental responsability in small and medium enterprises (SMEs)}

\section{ABSTRACT}

This document analyzes the social and environmental responsibility of small and medium-sized enterprises (SMEs) in Bogotá in order to identify the priorities of these organizations in relation to their contribution to the environmental and social improvement of the city. Corporate social and environmental responsibility should be understood as those responsible activities that go beyond compliance with business rules and legislation. Regardless of the size of the company, the lack of efficient social and environmental management can trigger problems such as the improper use of natural resources, environmental pollution, and low-quality jobs, among others. Data collected from surveys show that the SMEs located in Bogotá are concerned on Social and Environmental Responsibility, and carry out actions that they consider environmentally and socially responsible. However, they do not measure the impacts that their operation and commercialization generate, nor do they measure the impact of their social and environmental responsibility activities.

Keywords: Corporate Social Responsibility; Environmental responsability; SMEs; management. JEL: M10, M14, M19

\section{INTRODUCCIÓN}


Bogotá es la ciudad que presenta una mayor contribución a la creación de empresas del país, en el 2017, esta contribución representó, de acuerdo con la Cámara de Comercio de Bogotá (2018), un 22,5\% del total de empresas creadas y un incremento del $4 \%$ con respecto al año 2016. Indica la Cámara de Comercio de Bogotá (2018) que:

Con un total de 728.784 empresas y establecimientos comerciales al 31 de diciembre de 2017, Bogotá continúa siendo el principal centro de negocios del país, de este volumen, 663.285 son microempresas que corresponden al 91\%; 47098 son pequeñas representando el 6.5\%; 13.261 son medianas con un $1.8 \%$ y 5140 son grandes siendo el $0.7 \%$ del total. (p.34)

Cifras que han permitido reconocer el alto volumen de empresas cuyas operaciones, distribuidas entre las veinte (20) localidades de la ciudad, afectan directamente las condiciones ambientales y sociales de los pobladores. Los objetivos propuestos en esta investigación, estuvieron centrados en determinar cuál es nivel de relación entre el compromiso con la responsabilidad social y ambiental de las PYMES de Bogotá y, el compromiso con el impacto ambiental y social causado en la ciudad; lo cual, ha quedado calculado en las pruebas de coeficiente de correlación Rho Spearman (Universidad de Chile, 2014), que muestran una relación directa y significativa. También, se ha medido el nivel de conocimiento de la responsabilidad social y ambiental, las acciones y mediciones del impacto, que las PYMES desarrollan desde su gestión y su relación con los resultados ambientes y social de la ciudad.

Dentro de los resultados encontrados podemos observar que la gran mayoría las empresas encuestadas (79.84\%), no conocen con precisión los impactos ambientales producto de su operación y comercialización. La mitad de las empresas conocen que generan impactos ambientales en la ciudad, pero no los han medido; en cuanto al aspecto social un $60.90 \%$ de las empresas reconocen que su operación genera impactos sociales, pero tampoco los han identificado ni los han medido.

\section{REVISION DE LITERATURA}

\section{Responsabilidad social empresarial (RSE)}

Para fines de compresión de algunos términos, se ha abordado la responsabilidad social empresarial desde el concepto de la Comisión de las Comunidades Europea (CCE), en la cual se define:

La RSE es un concepto arreglo al cual las empresas deciden voluntariamente contribuir al logro de una sociedad mejor y un medio ambiente más limpio. Ser socialmente responsable no significa solamente cumplir plenamente las obligaciones jurídicas, sino también ir más allá de su cumplimiento invirtiendo en el capital humano, el entorno y las relaciones con los interlocutores. (p. 65)

Desde las diferentes posturas respecto a la RSE y su impacto, esta investigación parte de Lozano, J.M. (2009) bajo el concepto de la empresa ciudadana, la cual no constituye solamente una institución económica, sino también una institución social. Por ello, deben rendir cuenta de sus actividades de producción y comercialización con lo relacionado a lo económico, social y ambiental. Asimismo, debe estar pendiente a los impactos 
generados por su actuar productivo y comercial, desde su modelo de negocio (Lozano J.M., 2009).

Pequeñas y Medianas Empresas

La definición de PYMES está sometida a la Ley No. 590 de 10 de julio de 2000, que establece los siguientes parámetros:

1. Mediana Empresa: Planta de personal entre cincuenta y uno (51) y doscientos (200) trabajadores; Activos totales por valor entre cinco mil unos (5.001) y quince mil (15.000) salarios mínimos mensuales legales vigentes.

2. Pequeña Empresa: Planta de personal entre once (11) y cincuenta (50) trabajadores; Activos totales por valor entre quinientos uno (501) y menos de cinco mil unos (5.001) salarios mínimos mensuales legales vigentes.

3. Microempresa: Planta de personal no superior a los diez (10) trabajadores; Activos totales por valor inferior a quinientos uno (501) salarios mínimos mensuales legales vigentes.

Finalmente, con relación a los procesos administrativos y de gestión, Chanlat (2002) define a la gestión como un:

Conjunto de prácticas y actividades fundadas sobre cierto número de principios que apuntan a una finalidad: la búsqueda de la eficacia, sobre todo económica. Así, la gestión es para la empresa privada lo que la administración es para la empresa pública. (p. 32)

La situación de la Responsabilidad de la Empresa en las PyMEs de Latinoamérica y el Caribe revela que una gran mayoría de PyMEs latinoamericanas realizan acciones y actividades ligadas con la RSE a pesar de su desconocimiento formal del concepto en muchos casos. No obstante, sólo una pequeña parte de las empresas tiene un grado alto de implantación de estas actividades. Son precisamente las PyMEs más grandes, las manufactureras, las que cuentan con actividad exportadora y las que gozan de una buena situación económica las que presentan los mayores grados de implantación de la RSE (Vives et al.2005). Por otro lado, las Pymes colombianas se encuentran en una denominada etapa incipiente, donde los intereses económicos como la rentabilidad y la competitividad en términos de menores costos rigen las políticas de las empresas pequeñas y medianas que luchan por sobrevivir, lo cual es notorio en las principales ciudades colombianas, incluso en aquellas de menor tamaño (Flórez, 2013).

Sin embargo, estudios recientes para el caso de la ciudad de Medellín, indica que los empresarios aplican conocimientos, actitudes y prácticas de Responsabilidad Social, sin estar necesariamente asociados a su planeación estratégica. Lo anterior indica, que es necesario el fortalecimiento institucional con el fin de garantizar que estas organizaciones minimicen sus impactos negativos a la sociedad y trabajen por contribuir al desarrollo sostenible, sobre todo teniendo en cuenta que las actividades de producción de este sector contribuyen notablemente al deterioro del medio ambiente (Giraldo et al., 2015). Incluso, si se considera el sector de alimentos y bebidas, la pequeña empresa presenta un nivel de integración de principios y materias fundamentales de RS. Sin embargo, se evidencian brechas en la integración de la responsabilidad social, por lo que se hace 
necesario fortalecer el enfoque de RSE como un factor estratégico para la competitividad en el largo plazo (Ocampo et al., 2015).

Si se considera el sector financiero, que se caracteriza por estar conformado por entidades que pueden categorizarse como empresas grandes que establecen políticas de responsabilidad social, que incluyen factores económicos y sociales, ambientales, y que en cada uno de ellos dan cuenta de actividades y estrategias para cumplir con los lineamientos establecidos; tienden hacia una marcada inclinación a la dimensión económica, reflejada en el incremento desproporcionado de sus ingresos y utilidades en comparación con el de otros sectores productivo (Marulanda, 2014).

\section{METODOLOGIA}

El tipo de investigación es aplicado, siguiendo lo establecido para Murillo (2008), la cual se caracteriza por buscar la aplicación o utilización de los conocimientos adquiridos, a la vez que se adquieren otros, después de implementar y sistematizar la práctica basada en investigación. El uso del conocimiento y los resultados de investigación que da como resultado una forma rigurosa, organizada y sistemática de conocer la realidad. El nivel es correlacional, ello implicó un análisis del comportamiento de cada una de las variables escogidas, para luego efectuar una relación entre ellas; para lo cual se tiene en cuenta la apreciación de Hernández et al. (2010): "este tipo de estudios busca describir fenómenos, situaciones contextos y eventos, esto es detallar, como son y se manifiestan".

Por otro lado, el diseño de la investigación fue no experimental (puesto que la variable independiente no se altera), de corte transversal (puesto que la información se recaba en un momento del tiempo), definido por Hernández et al. (2010) como aquellos que tienen como objetivo describir variables y analizar la incidencia de las modalidades o niveles de una o más variables en una población en un momento dado, pues se ha realizado sin manipular deliberadamente las variables de estudio y solo se describió una realidad.

La determinación de los impactos se logró mediante la aplicación de herramientas de recolección de sobre las prácticas sociales y ambientales de las pymes en Bogotá y, el impacto causado en la ciudad como consecuencia de dichas prácticas. Para tal fin, se emplearon dos (2) instrumentos: Un cuestionario de autoevaluación para determinar los impactos ambientales y sociales en la ciudad; y otro, para determinar las responsabilidades sociales y ambientales de las Pymes. Ambos estructurados con preguntas cerradas con base a estudios previos (Ospina et al., 2003).

\section{RESULTADOS}

Existe una relación directa entre el compromiso de las PYMES de Bogotá con la responsabilidad social empresarial y, el compromiso con los impactos ambientales y sociales en la ciudad. Los resultados muestran que la variable compromiso con la responsabilidad social y ambiental de las empresas PYMES de Bogotá, obtuvo un valor medio general de 3,8617 con una desviación típica de 0,74516. Por lo tanto, las PYMES de Bogotá tienen un compromiso con calificación de nivel alto respecto a la responsabilidad social empresarial. 
La variable compromiso con el impacto ambiental y social en la ciudad, obtuvo un valor medio general de 2,64 con una desviación típica 0,7999. Por tanto, el nivel de compromiso que tienen las PYMES de Bogotá respecto al impacto ambiental y social en la ciudad es calificada con un nivel regular. En general, las empresas conocen los temas de responsabilidad social empresarial, realizan una planeación, indican que cuentan con programas de responsabilidad ambiental y conocen la legislación, dando una calificación de nivel alto al respecto de estas dimensiones. Igualmente, las empresas consideran que desarrollan actividades de responsabilidad ambiental y social, algunas de estas las califican como innovadoras y su nivel es alto.

Para la dimensión medición de los impactos de las actividades de responsabilidad social y ambiental, la calificación es de nivel bajo, es decir, las PYMES no miden los impactos que generan sus actividades de responsabilidad social y ambiental en la ciudad. Respecto al compromiso con los impactos generados en la ciudad, las PYMES indican que conocen los temas de los impactos ambientales y sociales, pero, no han medido los impactos que causan sus operaciones en la ciudad. Lo anterior indica que con relación a conocimientos la calificación es de nivel alto, pero en el caso de la medición del impacto, el nivel es bajo. En cuanto al conocimiento, la participación y el apoyo de los programas de RSE por parte de entidades público privadas, lo califican con un nivel regular.

Las empresas PYMES de Bogotá no cuentan con certificados de responsabilidad social y ambiental, por consiguiente, en su gran mayoría la calificación es de nivel bajo. Así como tampoco, se encuentra una calificación alta en procesos de producción limpia, donde la calificación es de nivel regular. Finalmente, para la dimensión interacción con los grupos de interés, los empresarios han calificado de nivel alto el conocimiento que tienen de los grupos de interés y de nivel bajo la interacción y programas con los estos grupos.

\title{
DISCUSION Y CONCLUSIONES
}

Los resultados obtenidos son coherentes con la evidencia disponible para el caso de la ciudad de Medellín. En este caso, Giraldo et al. (2015) señalan que:

\begin{abstract}
Alrededor del $57,7 \%$ de las empresas encuestadas reconocen y manifiestan aplicar conocimientos, actitudes y prácticas de Responsabilidad Social, sin estar necesariamente asociados a su planeación estratégica, por tanto, es necesario que estos elementos sean fortalecidos y mejorados para garantizar que estas organizaciones minimicen sus impactos negativos a la sociedad y trabajen por contribuir al desarrollo sostenible, sobre todo teniendo en cuenta que las actividades de producción de este sector contribuyen notablemente al deterioro del medio ambiente. (p. 45)
\end{abstract}

Toda vez que, la falta de una asociación de la responsabilidad social empresarial con la planeación estratégica, se puede relacionar con la falta de medición de las actividades de responsabilidad social y la falta de interacción con los grupos de interés. Esta situación es similar a la planteada para el caso de las pequeñas empresas del sector de alimentos y bebidas de Caldas, quienes tienen un mayor nivel de integración de principios y materias fundamentales de RS, pero que, sin embargo, se evidencian brechas en la integración de la responsabilidad social (Ocampo et al., 2015). 
Existe un gran avance respecto a la implementación de los programas de responsabilidad social en las empresas. En el año 2005, solo una pequeña parte de las empresas encuestadas, tenían un alto grado de implementación de actividades de RSE (BID, 2005). De acuerdo con los resultados de la presente investigación las empresas conocen, tienen programas y desarrollan actividades de responsabilidad social y ambiental. Por tanto, y para el caso de las PYMES en la ciudad de Bogotá, muestran un positivo avance.

A pesar de ello, es imperativo continuar esta línea de progreso, de tal manera que, las PYMES lleguen a la medición del impacto ambiental y social, que tanto su operación como su comercialización desencadenan en la ciudad. Así como también, medir el impacto que su gestión ambiental y social aporta a la ciudad, para poder determinar si dichas acciones de responsabilidad social, responden a las necesidades y requerimientos de la ciudad y sus pobladores.

Hasta tanto, las empresas no midan con precisión estos impactos en la ciudad, no podrán direccionar sus acciones de responsabilidad social empresarial hacia la mitigación de dichos impactos. De este modo, el conocimiento, los programas y las acciones que las empresas lleven a cabo, no responderán necesariamente, a resolver los problemas sociales y ambientales producto de su operación. Lo anterior indica que la sociedad en su conjunto debe buscar la manera de mejorar cada vez el uso de los recursos en los procesos de producción y fabricar productos más eficientes, que demanden una menor cantidad de energía. Lo anterior debe convertirse como parte integral de las prácticas industriales habituales dado el rol de las empresas en la producción de CO2 (ONU, 2004).

De otro lado, la interacción con los grupos de interés es la puerta de entrada a la relación ciudad - empresa. Si el sector de las PYMES no identifica de manera clara las necesidades y requerimientos de sus grupos de interés, no podrán aportar acertadamente a la mitigación de los impactos causados en la ciudad. No es suficiente que las empresas reconozcan los grupos de interés, sino que deben relacionar su gestión de RSE con estos grupos.

\section{REFERENCIAS}

Cámara de Comercio de Bogotá (2018). Informe de gestión al 31 de diciembre de 2017.

Comisión de las Comunidades Europeas (2001). Libro verde. Fomentar un marco europeo para la responsabilidad social de las empresas. Bruselas. Recuperado de: http://www.europarl.europa.eu/meetdocs/committees/deve/20020122/com(2001)366 es.pdf

Días, D. P. \& Hernández, B. E. (2016). Responsabilidad Social Empresarial y análisis de Comercio Justo en la fruta Camu-Camu en Tarapacá-Amazonas. Universidad de la Salle.

Flórez, A. P. (2013). La responsabilidad social empresarial en las pymes de Colombia - Análisis a resultados de aplicación de Herramienta de Caracterización y Manual Cartilla para pequeños y medianos empresarios. Universidad Escuela de Administración de Negocios.

Giraldo, L. M., Kammerer, Y.Y., Rios, L. A. (2015). Responsabilidad social en pymes del área metropolitana de Medellín, Colombia. Dimensión Empresarial, 14(1), 123-135.

Hernández Sampieri, R., Fernández Collado, C., Baptista Lucio, P. (2015). Metodología de la Investigación. México: McGraw Hill.

Lozano, J. M. (2009). La empresa ciudadana como empresa responsable y sostenible. Madrid: Editorial Trotta.

Marulanda, P. A. (2014). Prácticas de responsabilidad social empresarial de la banca colombiana: estudio documental sobre Bancolombia, Banco de Bogotá y Davivienda.

Murillo, W. (2008). La investigación científica. Recuperado de: http://docencia.fca.unam. mx/ mvazquez/archivos/WebquestTMCSIcientifica.pdf 
Ocampo, O., García, J., Forero, Y. (2015). Responsabilidad social en pequeñas empresas del sector de Alimentos y Bebidas de Caldas. Entramado, 17(2), 72-90. http://dx.doi.org/10.18041/

Organización de las Naciones Unidas para la Ciencia y la Cultura (2004). Manual sobre diversidad y estilos de vida. Jóvenes por el cambio. Recuperado de https://www.portalciudadano.cl/wpcontent/uploads/2015/01/ManualConsumo.pdf

Ospina, B., Sandoval, J., Aristizábal, C., Ramírez, M. (2003). La escala de Likert en la valoración de los conocimientos y las actitudes de los profesionales de enfermería en el cuidado de la salud. Investigación y Educación en Enfermería, 23(1), 15-16.

República de Colombia (2000). Ley 590 de 10 de julio de 2000. Imprenta Nacional de Colombia.

Vives, A., Corral, A., Isusi, I. (2005). Resumen ejecutivo. Responsabilidad Social de la Empresa en las PyMEs de Latinoamérica. Banco interamericano de desarrollo e Instituto Vasco de estudios e investigación. Washington, D. C.: BID 